
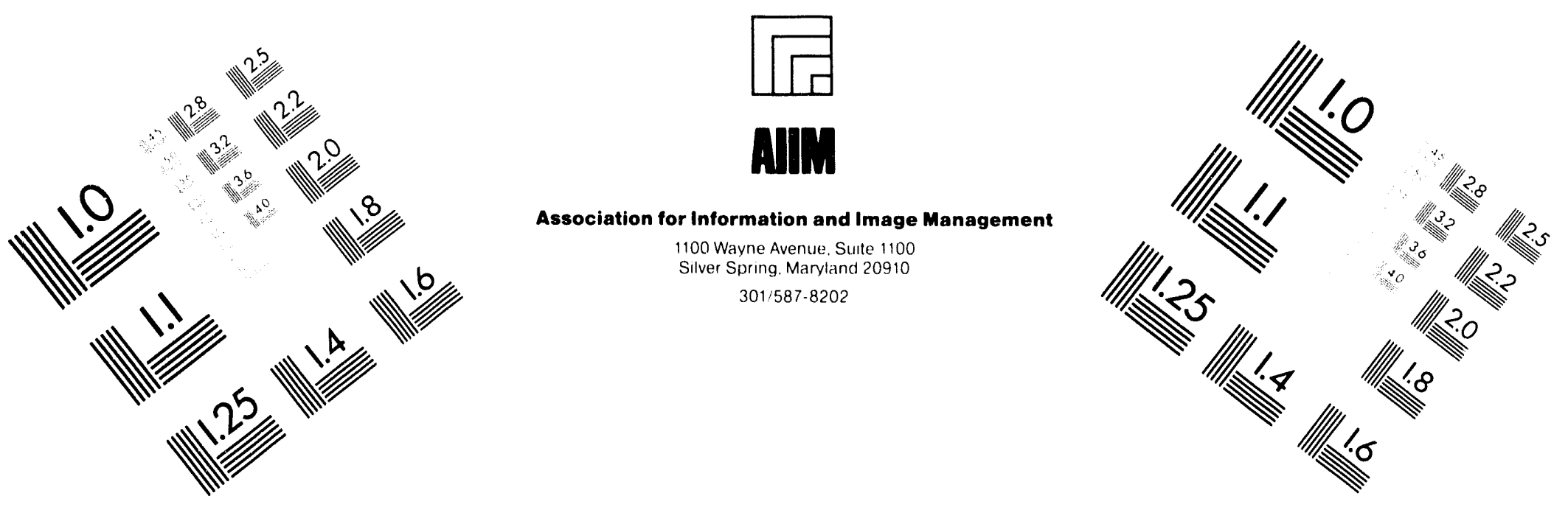

\title{
Centimeter
}

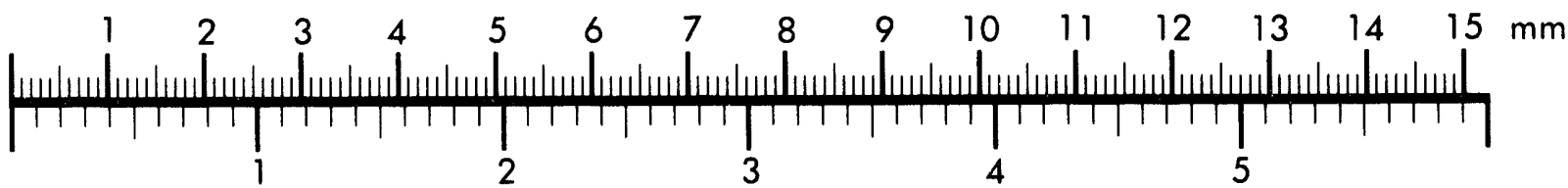

Inches
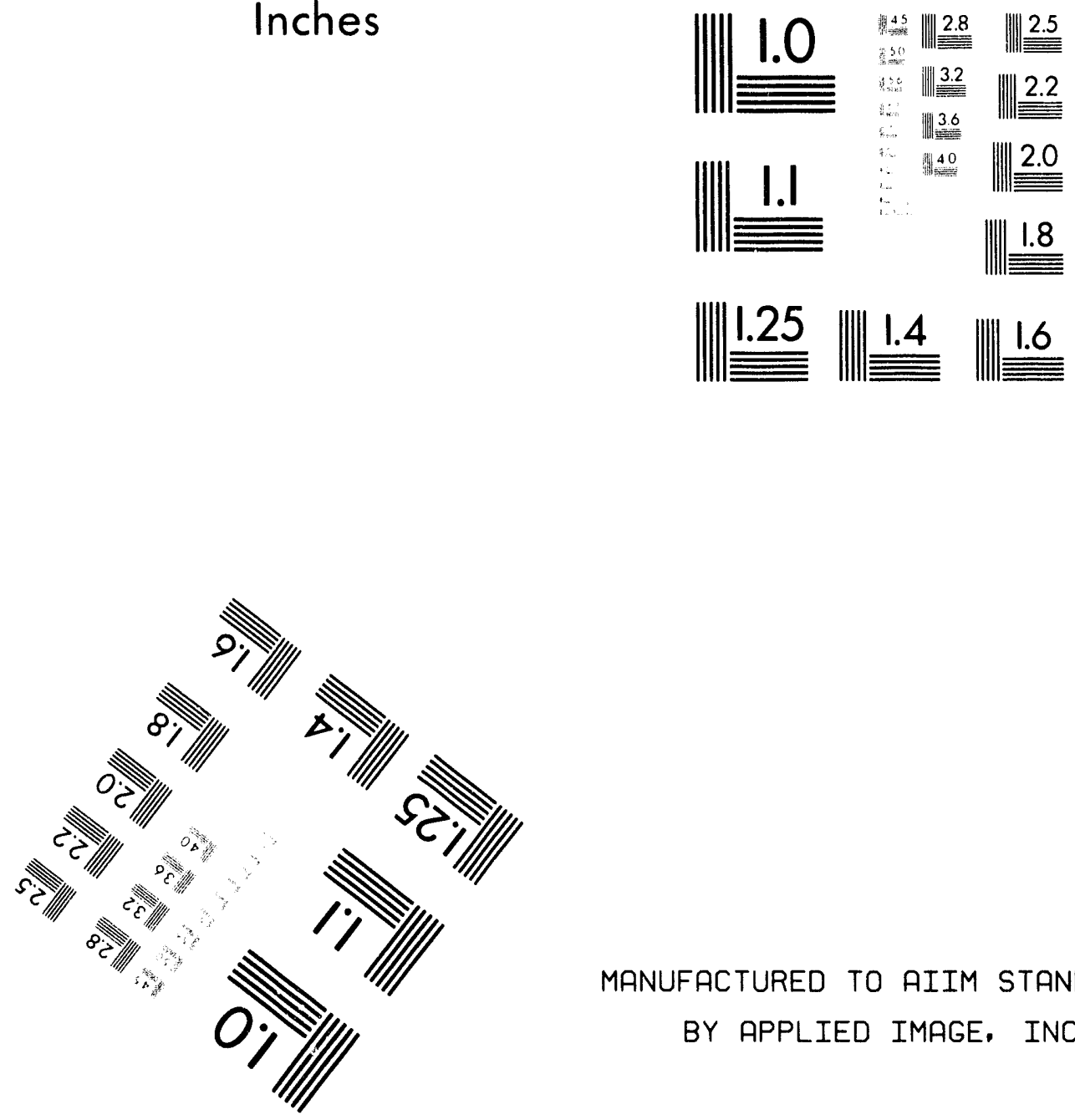

MANUFACTURED TO AIIM STANDARDS

BY APPLIED IMAGE, INC.

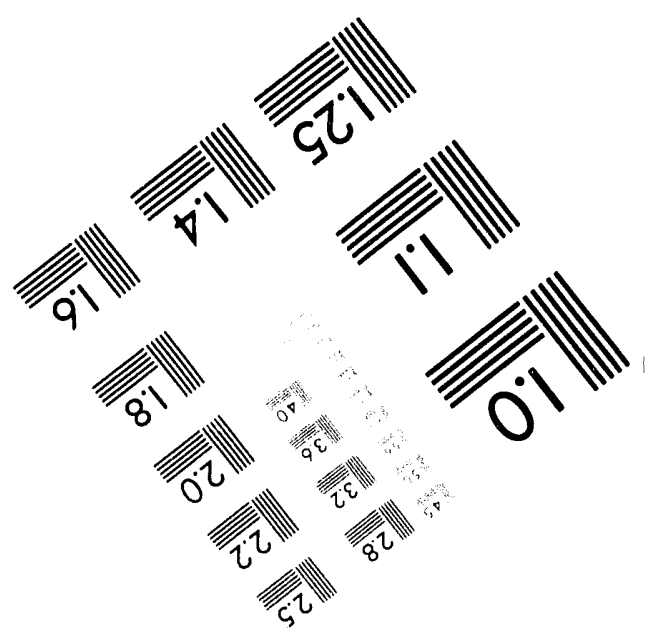



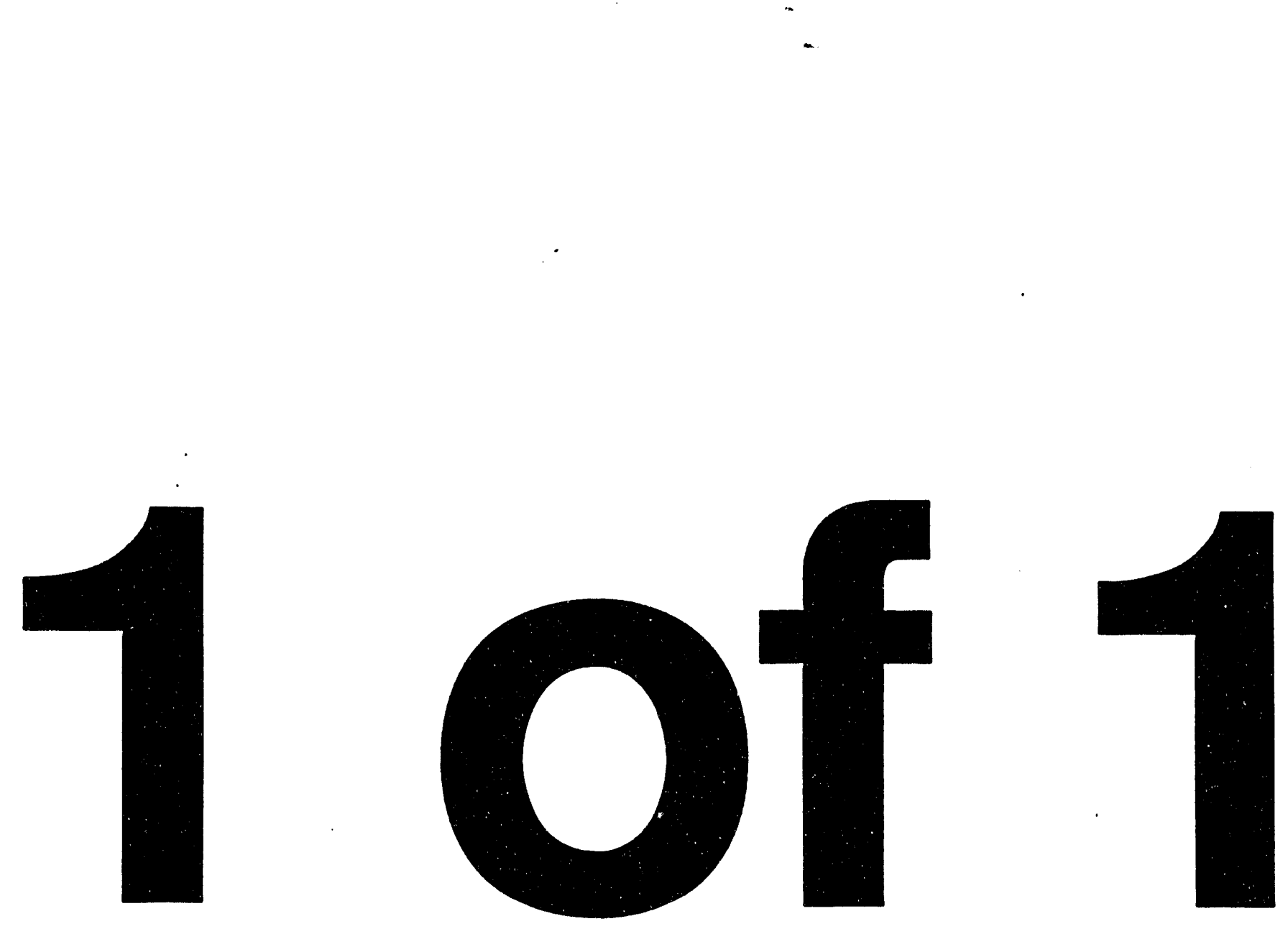


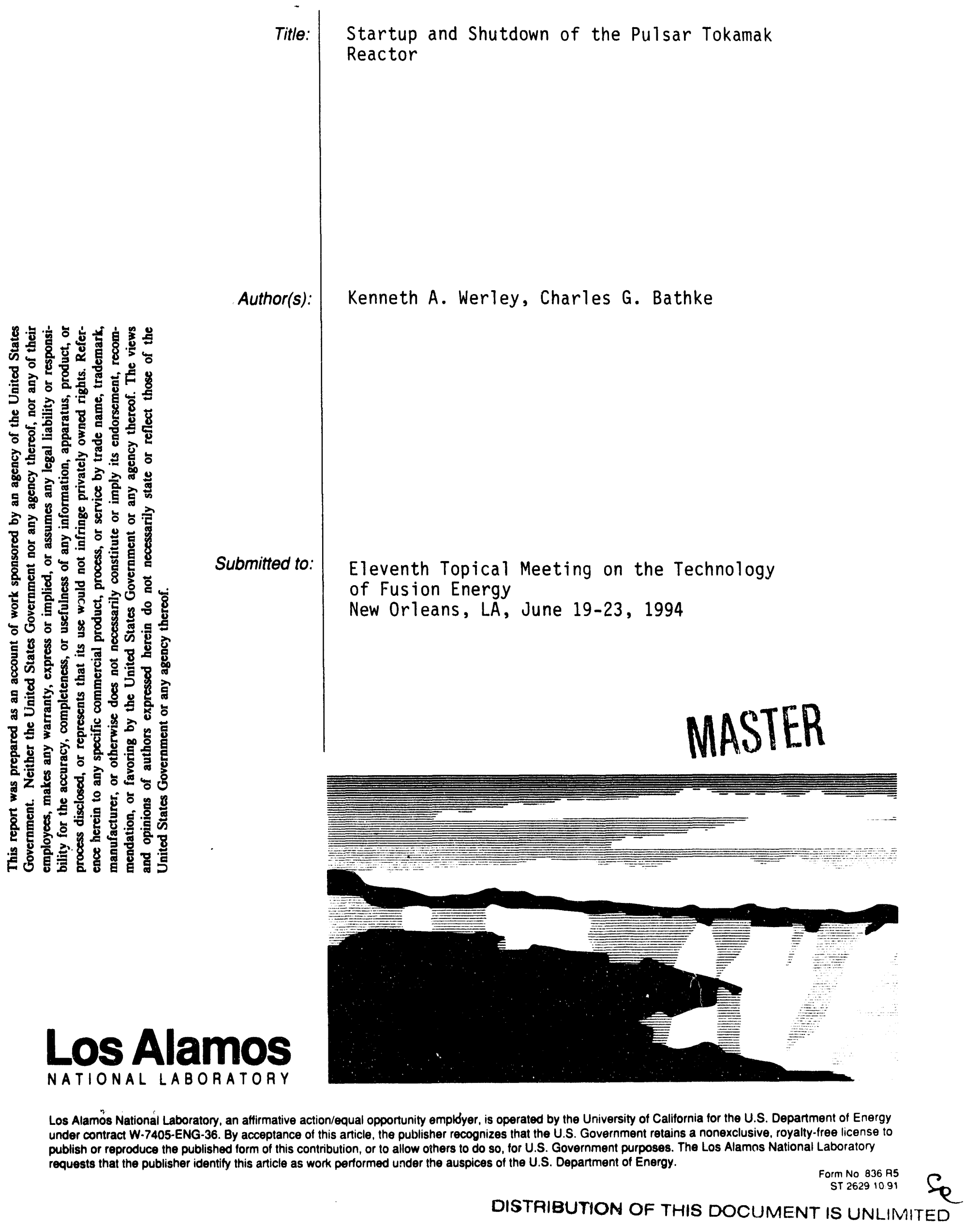




\section{STARTUP AND SHUTDOWN OF THE PULSAR TOKAMAK REACTOR ${ }^{\dagger}$}

\author{
K.A. Werley \\ Los Alamos National Laboratory \\ P.O. Box 1663 \\ Los Alamos, NM 87545, USA \\ (505)667-3680
}

\author{
C.G. Bathke \\ Los Alamos National Laboratory \\ P.O. Box 1663 \\ Los Alamos, NM 87545, USA \\ (505)667-7214
}

\begin{abstract}
Start-up conditions are examined for a pulsed tokamak reactor that uses only inductive plasma current drive for startup, burn and shutdown. A zerodimensional (profile-averaged) model that describes plasma power and particle balance equations is used to study several aspects of plasma startup and shutdown, including optimization of the startup pathway tradeoff of auxiliary startup heating power versus startup time, volt-second consumtion, thermal stability and partial-power operations.
\end{abstract}

\section{INTRODUCTION}

Following the Advanced Reactor Innovation and Evaluation Study (ARIES ${ }^{1}$ ) of steady-state tokamaks is the PULsed Advanced Reactor (PULSAR ${ }^{2}$ ), which features pulsed operation. The PULSAR-I design ${ }^{2}$ has a plasma current of $I_{\phi}=13.2 \mathrm{MA}$, a major radius of $R=8.5 \mathrm{~m}$, a minor radius of $a=2.13 \mathrm{~m}$, and an average net electric power of $1 G W e$. The internal heat capacity of the shield can provide up to $200 \mathrm{~s}$ of quality heat before external thermal storage would be required. Thus, a downtime of $200 \mathrm{~s}$ between fusion power pulses is desired to avoid purchasing expensive external thermal storage capacity. Fig. 1 illustrates a time history of a single PULSAR pulse for the plasma current and fusion power. The downtime includes plasma shutdown, transformer recharge, and plasma startup. It is crucial to minimize the startup and shutdown times in order to allow the recharge times of a minute (or longer) to reduce recharge costs.

†This work was supported by the U.S. Department of Energy under Contract No. W-7405-ENG-36.
The present study examines the current risetime, the ignition time, and the plasma shutdown time, to find optimum startup and shutdown pathways that for a given auxiliary heating power take the shortest time. This startup time versus heating power trade-off information is given to the PULSAR systems code $\left(\mathrm{ASC}^{1}\right)$ for determining cost-optimized reactor designs. Additional startup issues include the voltseconds consumed, the divertor loadings, disruption and electron-run-away density limits, and operation at partial power levels for meeting reactor plant liscencing and load-following requirements.

Two downtime scenarios are considered. The baseline scenario terminates the plasma and the plasma current between fusion pulses. The second scenario, called the hybrid, seeks to reduce cyclic fatigue, power-supply, and PF-coil volt-second requirements by maintaining $l_{\phi}$ at nearly the full level between fusion pulses in a low-density plasma where rf current drive can work efficiently. Section II describes the startup model. Section III presents plasma operating-space contours and startup results for the basecase, and Sec. IV describes the hybrid startup and shutdown scenarios. Section V summarizes the important startup and shutdown issues.

\section{ZERO-DIMENSIONAL PLASMA MODEL}

A plasma model is used to calculate plasma operating contours and startup trajectories. The model includes ion and electron energy balance; protium, deuterium, tritium, helium-3 and helium4 (alpha) particle continuity; a specified ionic fraction for high-Z impurities; charge balance; and magnetic equilibrium constraints. An oxygen fraction of $1 \%$ is included in all simulations, and a variable xenon 


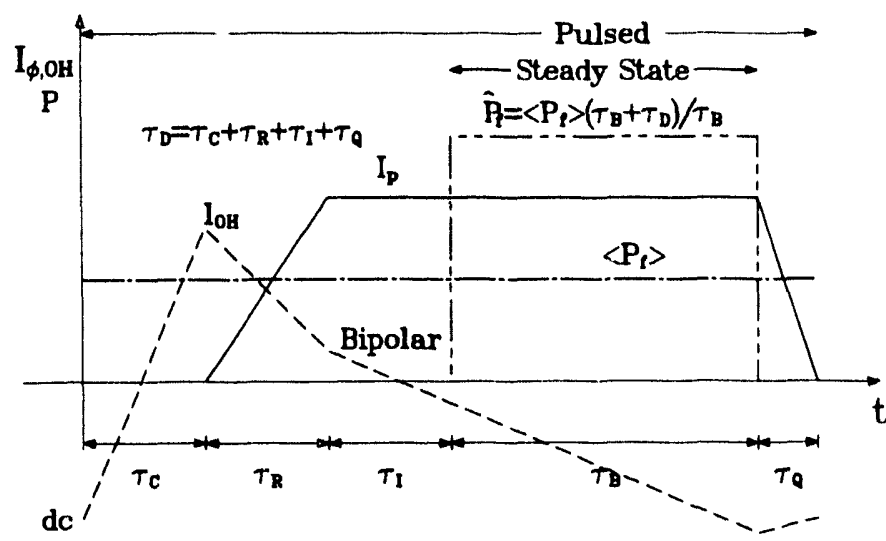

Fig. 1. A schematic of a PULSAR single-pulse time history. The time-period subscripts, e.g. $\tau_{C, R, I, B, Q, o r D}$, identify the coil Charge (C), plasma current Rise (R), Ignition (I), plasma Burn (B), plasma current Quench (Q), and the total Downtime between burns (D).

fraction is added to model the PULSAR hybrid case. The plasma physics model ${ }^{3,1}$ is similar to that contained in the ARIES System Code (ASC).

The ion and electron energy-balance equations include fusion, auxiliary and ohmic heating, electronion equipartition, transport, bremmstrahlung, cyclotron and line radiation. The fractional fusion power deposited in the ions is calculated through a time integral over the slowing down time of the slowing down rate on the ions; zero prompt loss is assumed. Cyclotron radiation loss is a function of the effective wall reflectivity, $R_{C Y C}$, which includes the effects of holes in the wall. The fractional auxiliary power going to the ions is $f_{A U X_{i}}=0.05$, and the auxiliary power, $P_{A U X}$, must be specified.

All background ions are assumed to share the same local temperature, $T_{i}(r)$. The total ion density, $n$, the electron density, $n_{e}$, (assuming charge neutrality) and the effective ionic charge, $Z_{\text {eff }}$, are obtained by summing over the ion species. The average $Z$ and $Z^{2}$ of impurity species and line radiation are calculated assuming coronal equilibrium. All ion species are assumed to have the same particle confinement time, $\tau_{p}$. The ratio of $\tau_{p} / \tau_{E}=4$, must be specified. Edge fueling is assumed, so the fueling time is limited to be no shorter than $\tau_{p}$. The plasma energy confinement time, $\tau_{E}$, is modeled by an inverse quadrature relationship between neo-Alcator and a multiple of ITER- $89 p^{4}$ confinement times. This confinement multiplier is given by $H=1$, for $\mathrm{L}$ mode operation, and 2.48 for $\mathrm{H}$-mode. The transition from $\mathrm{L}$ to $\mathrm{H}$ is assumed to occur when the transport separatrix heat flux exceeds a threshold given by $q^{\text {thresh }}=4 \times 10^{-16} n B$, in mks units. The (reverse)
$\mathrm{H}-\mathrm{L}$ transition is assumed to occur at $0.5 q^{\text {thresh }}$. The ratio of $\tau_{E} i / \tau_{E} c$ is taken to be 0.5 . The equilibrium is defined through the specification of $I_{\phi} / a B$, elongation, $\kappa=1.8$, triangularity, $\delta=0.5$, and the inverse aspect ratio, $\epsilon=0.25$. To complete the 0 -D description, plasma profiles for $n, T e$, and $J$ are specified, and profile form factors are calculated.

This plasma model is incorporated irito both a steady-state and a time-dependent numerical code. The steady-state MakPOP code surveys all operating space and plots contours of constant $P_{A U X}$. The time-dependent model, BURN, adds a Fokker-Planck formalism to describe the time-dependent slowing down of the charged-particle fusion products. The plasma initial conditions are $I_{\phi}(0)=0.25 \mathrm{MA}$ and $T_{e}(0)=T_{i}(0)=0.4 \mathrm{keV}$. The initial plasma density is constrained so that the initial streaming parameter, which is the ratio of the electron drift speed to the thermal speed, has a value of 0.2 . Above this value, the electric fields would generate significant numbers of run-away electrons.

One concern during startup is that plasma-wall interactions may be a source of impurities that will degrade the main plasma. To monitor the potential for sputtering erosion, the analytic high-recycle divertor model of Cooke ${ }^{1,5}$ is applied. This model estimates the peak divertor-plate heat flux and the peak plasma temperature. The model has been benchmarked against 2-D edge-transport codes for the ARIES I operating-point. ${ }^{1}$ The divertor model is not expected to describe early startup accurately, and is used only to gauge the relative loading compared to a steadystate high-recycle divertor.

\section{INDUCTIVE STARTUP BASECASE}

To identify the optimal startup path, heating contours in $(n, T)$-space were constructed around the PULSAR burn point, and are illustrated in Fig. 2. The optimal path passes through the local auxiliary heating minimum, including the saddle point. The minimum power to ignite is $\sim 24 \mathrm{MW}$. Increasing $P A U X$ above the minimum ignition value shortens the startup time required to traverse the optimal path. The thermal stablility properties of the potential operating space are also contained in Fig. 2. Stability occurs in those regions where constant- $n$ positive- $T$ perturbations require increased $P A U . X$ to maintain power balance. A thermally stable PULSAR operating point is shown. The $P A U X$ contours also show that thermally stable partial-power operation can be achieved anywhere along the startup path. 


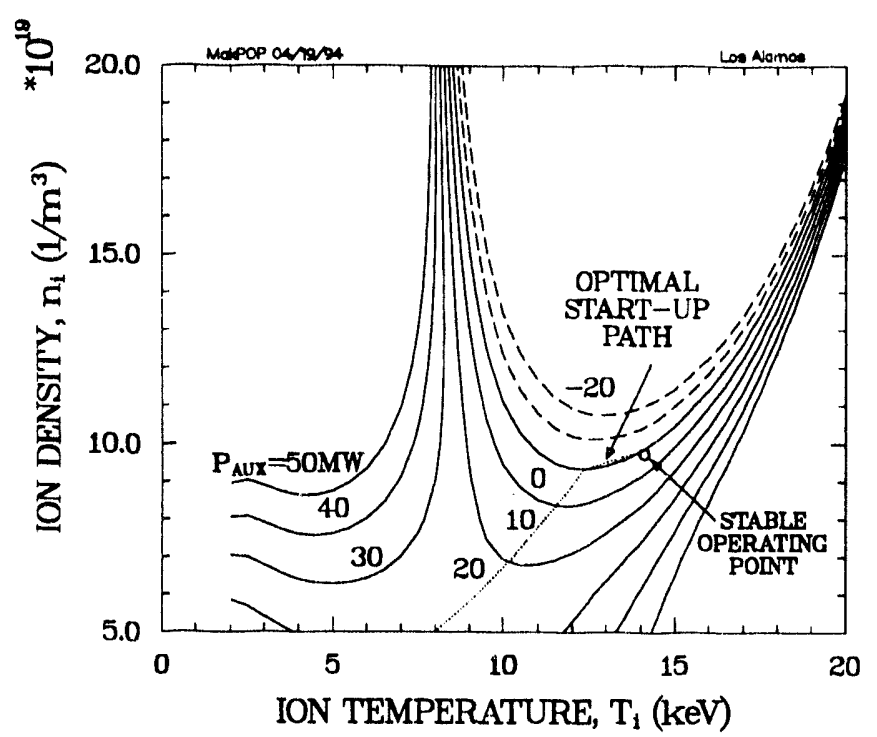

Fig. 2. PULSAR I plasma operating space auxiliary heating contours for a plasma with $I_{\phi}=13.2 M A, B=6.66 \mathrm{~T}$, $H=2.48, a=2.125 \mathrm{~m}$ and $R=8.5 \mathrm{~m}$.

Having identified the optimal ignition pathway, time-dependent simulations were performed. The plasma fueling algorithm is adjusted to follow the optimal pathway, subject to the constraint that fueling may not raise $n$ at a rate faster than particle transport allows. The first phase of startup ramps $I_{\phi}$ to its full value of 13.2 MA in about $30 \mathrm{~s}$. A maximum $0.5 \mathrm{MA} / \mathrm{s}$ ramprate is imposed based upon stability considerations of the time required for edge currents to diffuse into the plasma center. No auxiliary heating is used during this phase, and the density is controlled to avoid electron runaway production, to avoid crossing the density disruption limits, to have low edge temperatures to avoid impurity generation, and to hold $T e$ to less than $2 \mathrm{keV}$ so that the edge plasma current will diffuse into the center more quickly. All this is done under L-mode confinement conditions.

After the current has reached full value, $P A U X$ is applied to achieve an $\mathrm{L}-\mathrm{H}$ transition. The $\mathrm{L}-\mathrm{H}$ heating requirement is greater than the heating needed to traverse the ignition boundary saddle point. After achieving $\mathrm{H}$-mode, particle fueling is increased to raise $n$ and achieve ignition. The auxiliary heating is adjusted to first achieve $\mathrm{H}$-mode, and secondly to assure that the Borass ${ }^{6}$ disruption density limit is not exceeded. Once the plasma crosses the ignition boundary, the auxiliary heating is turned off.

The temporal history of $n$ and $T$ is plotted in Fig. 3, starting after full current is achieved $(t=30 \mathrm{~s})$. Significant $P_{A U X}$ is applied at about $38 \mathrm{~s}$, and the transition to $\mathrm{H}$-mode occurs at $44 \mathrm{~s}$. The ignition boundary is crossed at $68 \mathrm{~s}$, and after that, the density is increased at a much slower rate to prevent a large

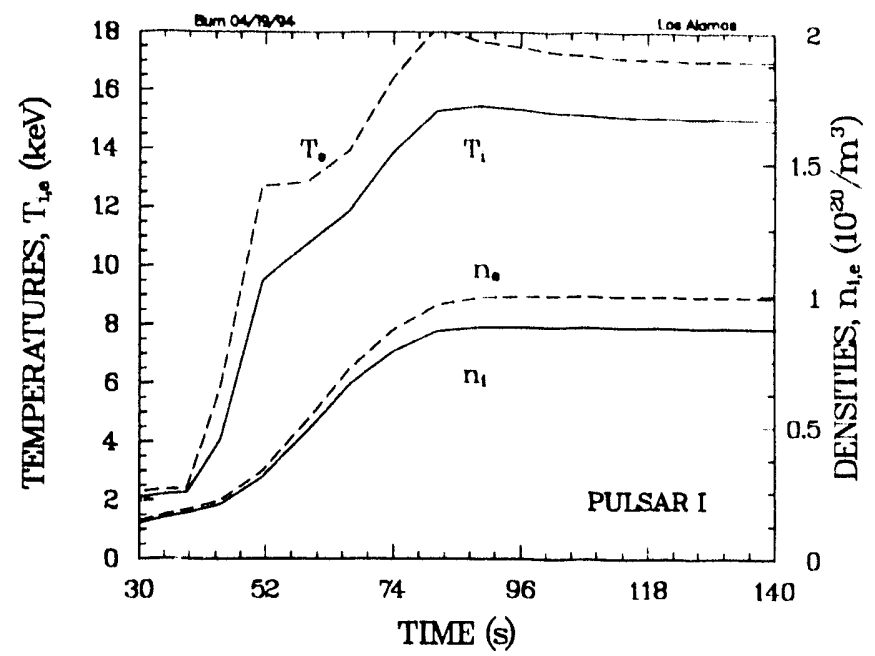

Fig. 3. PULSAR I basecase startup history for temperature and density.

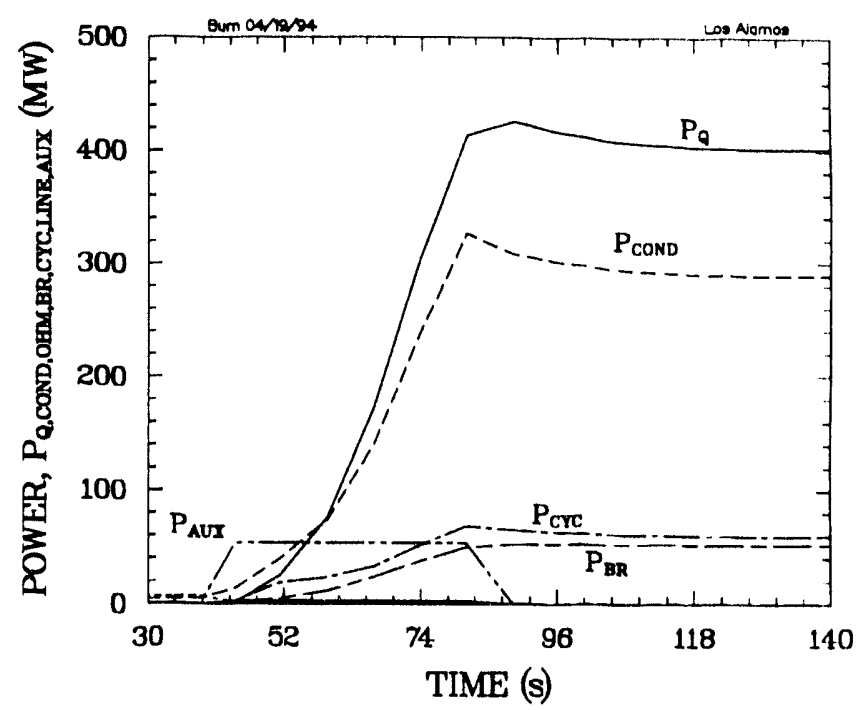

Fig. 4. ARIES IV plasma powers during inductive startup. Loss terms include the transport conduction, COND, bremmstrahlung, BR, and cyclotron radiation, CYC, powers. Heating terms include the fusion power carried by charged fusion products, $Q$, the ohmic heating, OHM, and the auxiliary heating power, AUX.

ignition fusion over-power surge, which if fueling is not carefully controlled can easily reach $70-80 \%$ above the steady operating point power. By slowly increasing the ignition density to the operating point value the over-power event is limited to only $5 \%$. The temporal history of the plasma powers are contained in Fig. 4. A minimum $P_{A U X}$ of $57 \mathrm{MW}$ is required to make the L-H transition.

It is fortunate that the H-L transition occurs at a factor of two lower transport power than the L-H transition. Simulations that set the H-L and L-H transitions equal would first make an $\mathrm{L}-\mathrm{H}$ transition 


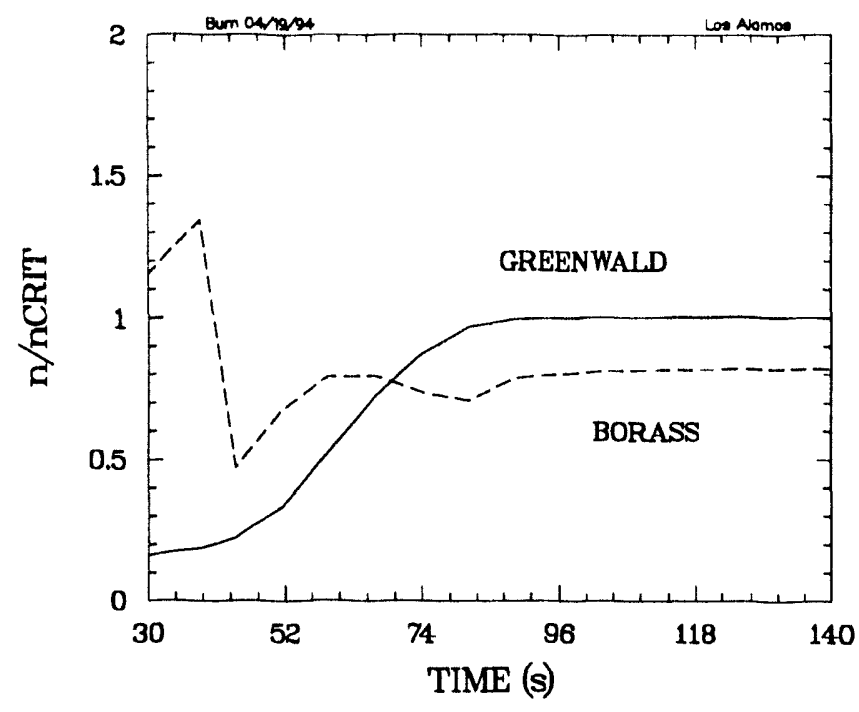

Fig. 5. PULSAR I bascase relative critical density for density. driven disruptions during startup.

at low density, but, as the density was raised, would convert back to L-mode before the ignition boundary was reached. Subsequently, auxiliary heating powers of 250-300 MW would be required to convert back to $\mathrm{H}$-mode and to reach ignition.

The cyclotron radiation losses shown in Fig. 4 are larger than Bremsstrahlung because of low siliconcarbide wall reflectivity. Adding a high reflectivity coating to the wall could reduce $I_{\phi}$ or the $\mathrm{H}=2.48$ value.

The ratios of the plasma density to the critical disruption density limits are shown in Fig. 5. The Greenwald ${ }^{7}$ constraint limits the average density, and the Borass $^{6}$ constraint limits the separatrix density. Both limits are marginally satified during startup. The Borass limit is particularly difficult to satisfy, since any out-of-phase change in the auxiliary heating or the fueling rate can easily push the density significantly above the Borass limit. The ITER CDA design ${ }^{4}$ allowed $125 \%$ and the ARIES designs ${ }^{1}$ permitted $200-400 \%$ of the Greenwald density limit.

The peak divertor $T e$ and heat flux on the plate is shown in Fig. 6 for a high-recycle divertor model. The heat flux is maintained at low values throughout startup, but high $T_{e}$ is estimated at the plate. The proposed PULSAR divertor is an advanced gaseous divertor which should better (than high recycle) protect the divertor. Figure 6 shows that the more advanced divertor operation must begin soon after full current is reached, and before high $n$ is attained.

In conclusion, the PULSAR I startup is simpler than some of the ARIES designs where profile control,

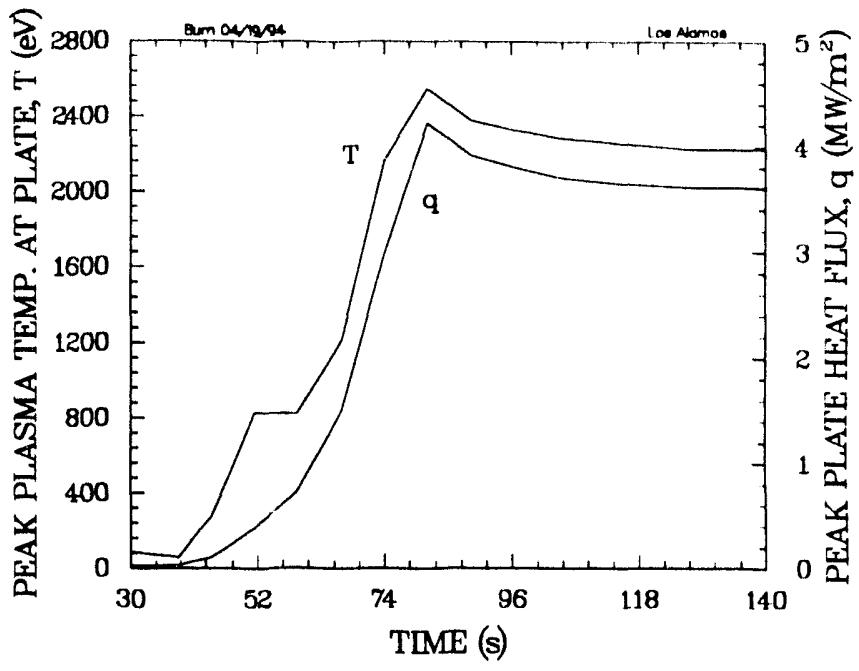

Fig. 6. PULSAR I bascase peak plasma temperature and density at the divertor plate for high-recycle divertor conditions.

second-stability limitarions, and rf-driven current ramp complicated startup. The PULSAR simulations also incorporate more physical reality than the earlier ARIES designs by enforcing $\tau_{E i} / \tau_{E e}=0.5$, that disruption density limits be satisfied, and that L-H transition power requirements be taken into account. The PULSAR I startup requires $57 \mathrm{MW}$ of auxiliary power and takes 84 seconds, of which the first 30 s are for the current ramp. Only 22 resistive voltseconds are consumed, which is less than a tenth of the inductive volt-seconds required.

\section{HYBRID STARTUP AND SHUTDOWN}

The hybrid mode maintains the plasma current continuously, even though the fusion burn is pulsed and limited by resistive volt-second consumption. Between burn pulses, the plasma density is reduced to a low value where rf current drive works more efficiently, and can be used to recharge the transformer for the next pulse. Plasma conditions where the recharge phase is sufficiently short ( $\sim 1$ minute) are difficult to achieve, and require a low density plasma with very poor total energy confinement. The plasmaoperating-contour model was used to identify plasma conditions where fast recharge could be accomplished. A range of impurities were examined, including $O$, $\mathrm{Fe}, \mathrm{Kr}$ and $\mathrm{Xe}$. Higher-Z impurities achieve higher radiation powers with lower concentrations and $Z_{\text {eff }}$, and achieve a given recharge time with lower rf powers. Short recharge times are achieved in plasmas with $0.2-0.4 \mathrm{keV}$ temperature and $1-2 \times 10^{19} / \mathrm{m}^{3}$ density. High- $\mathrm{Z}(\mathrm{Xe})$ impurity line radiation is needed with a density fraction of 0.0012 , a radiation fraction of $99 \%$ and $Z_{\text {eff }}=2.1$. These plasmas are thermally stable in at least a global (0-D, profile-averaged) sense 
using a coronal equilibrium line radiation model. Addressing thermal stability issues on a local scale was beyond the scope of this project.

This section examines the startup and shutdown scenarios that connect the burn conditions to the hybrid recharge phase conditions. These simulations are performed about an interim PULSAR operating point which had $I_{\phi}=11.5 \mathrm{MA}, H=2.17$, and $n_{i}=$ $1.4 \times 10^{20} / \mathrm{m}^{3}$, and the L-H and H-L transition was assumed to occur at a plasma heating power of $20 \mathrm{MW}$. Two cases were considered for the plasma current during the recharge phase. The first is a full current case (11.5 MA), which maintains the inductively supplied poloidal flux. The second has 7.3 MA, and the current is allowed to decrease, along with the plasma beta, during the shutdown period. The lower current case does not reduce the recharge current-drive power requirements significantly, because of changes in the plasma resistance. Also, the lower current case has a much smaller thermally stable recharge regime.

The hybrid startup differs fron the basecase PULSAR startup in two major ways. First, the plasma current need not be ramped up from zero, so the ramptime can be eliminated or reduced. Secondly, the hybrid plasma startup begins with a large high-Z impurity fraction that is radiating $99 \%$ of the power, so that plasma heating cannot begin until pumping reduces the impurity density. Since pumping can continue and impurities can be removed while startup is proceeding, the time saved by not ramping the current is the dominant effect. The hybrid startup time is about 56s for the full current case, and about 60s for the 7.3 MA case. This compares to the PULSAR basecase startup time of 84s. Hybrid startup histories are similar to basecase startup results. Disruption density limits are satified. An ignition heating power of only $40 \mathrm{MW}$ was needed in this hybrid simulation because of the (older) lower $(20 \mathrm{MW}) \mathrm{L}-\mathrm{H}$ transition assumption. The $Z_{\text {eff }}$ value decreases from 2.1 during the recharge phase to 1.6 at the operating point conditions.

It is also important to minimize the hybrid shutdown time. The main goal is to reduce the plasma density to the required recharge value. Impurity injection is needed to achieve high radiation fractions and low $T_{e}$ so that the recharge time can be small. It turns out that injecting impurities too soon causes taup to increase significantly resulting in longer shutdown times. The shutdown algorithm is, then, to pump particles as quickly as they diffuse from the main plasma, and inject the xenon impurities after the recharge density is reached. The shutdown time histories for the hybrid case that ramps the current

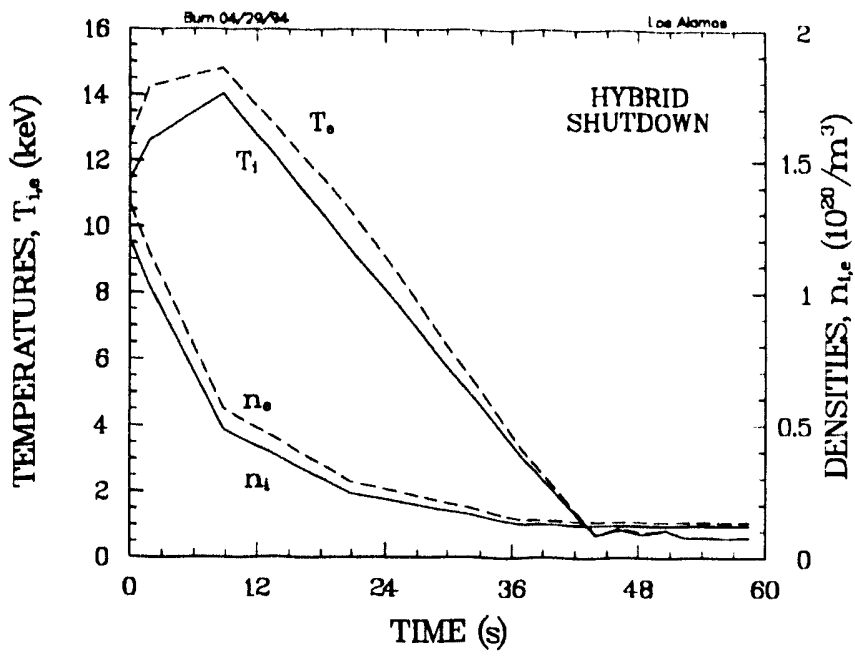

Fig. 7. PULSAR hybrid (partial current) shutdown trajectories of plasma temperature and density.

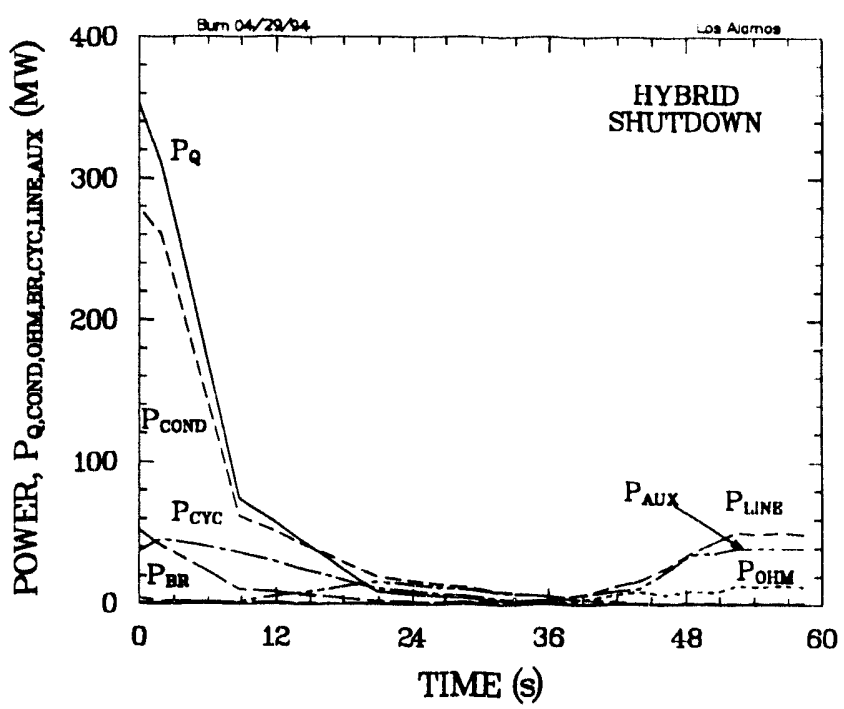

Fig. 8. PULSAR hybrid (partial current) shutdown trajectories of plasma power.

down from 11.5 MA to 7.3 MA are shown in Figs. 7 $\& 8$. The plasma current is ramped down at a rate of $0.5 \mathrm{MA} / \mathrm{s}$, but this rate could be as low as $0.1 \mathrm{MA} / \mathrm{s}$ without increasing the shutdown time.

As the plasma density initially decreases, the plasma temperatures first rise, because the plasma energy is discributed over fewer particles, until the density decrease becomes sufficiently large to lower the fusion power significantly. Because the fusion power is proportional to $n^{2}$, fusion shuts off quickly (in $10 \mathrm{~s})$ as $n$ drops. As the fusion heating becomes small, auxiliary heating must be added to prevent the Borass density limit, which is a function of the conduction power, from being exceeded by a factor of 5-6. A $P_{A l} \cdot x$ of $20 \mathrm{MW}$ is required to prevent the formation of an over-dense plasma. A concern existed that adding 


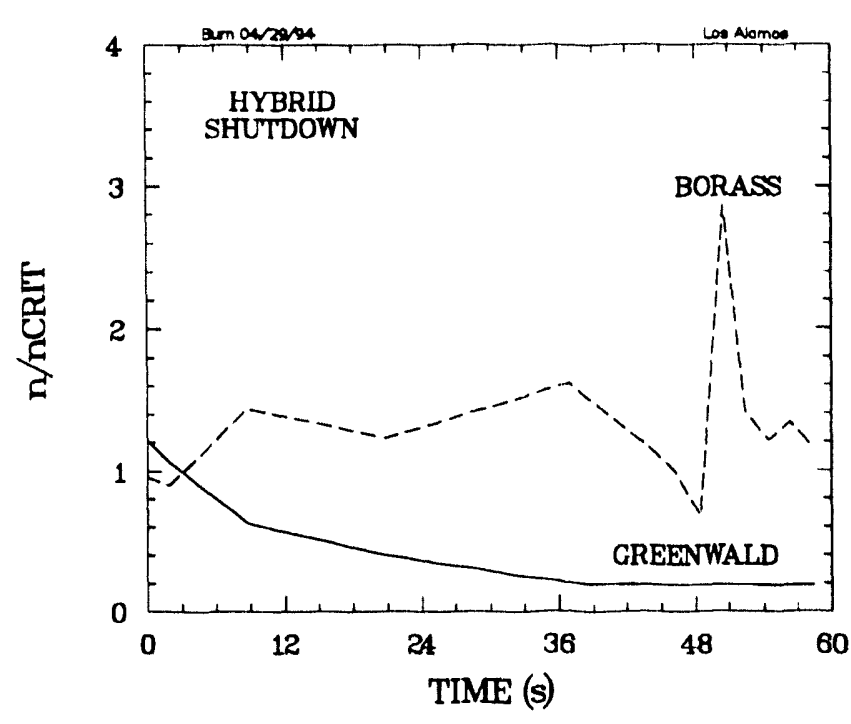

Fig. 9. PULSAR hybrid (partial current) relative critical density for density-driven disruptions during shutdown.

$P_{A U X}$ during the shutdown phase would extend the shutdown time, $\tau_{Q}$, by maintaining the plasma in $\mathrm{H}$ mode, with a longer $\tau_{p}$. The confinement time is also a function of $P_{C O N D}^{-0.5}$, which nearly cancels the effect of remaining in $\mathrm{H}$ mode, so that only a minor change occurs in $\tau_{Q}$. The critical density ratio is illustrated in Fig. 9. During the first 45s, controlling $P_{A U X}$ allows $n / n$ BORASS to be held near unity.

At about 42s, xenon is injected. Once the radiation fraction becomes large, the critical density limit becomes very sensitive to small perturbations in impurity density or in heating power, such that $n / n^{B O R A S S}$ can easily reach infinity. Very careful control of density, impurity fraction, and $P_{A U X}$ must occur while the recharge current drive is being turned on. In this simulation, the numerical algorithm allowed one 'little' spike where $n / n^{B O R A S S}$ reaches three. Improving the numerical algorithm to take smaller timesteps and to adjust $P_{A U X}$ more quickly would remove the spike. Never-the-less, in an actual experiment strong feedback control will be necessary.

As the fusion power decreases, the confinement time dependence on the square root of $P_{C O N D}$ causes $\tau_{p}$ to increase by a factor of 2-3 over the fusion burn conditions. This increase results in longer pumpout times than one might expect based upon the burn phase $\tau_{p}$ of $8 \mathrm{~s}$. The total hybrid shutdown time is $60 \mathrm{~s}$ for the full current case, and $52 \mathrm{~s}$ for the 7.3 MA case. Thus, if a maximum of $200 \mathrm{~s}$ of downtime is available between each fusion pulse, then about $84 \mathrm{~s}$ remain for the recharge phase. The basecase startup resistive poloidal flux consumption is $22 \mathrm{Vs}$, and the hybrid startup consumption is $10 \mathrm{Vs}$. The hybrid consumption is less because the plasma need not be maintained in the low $T_{e}$, high resistivity state for a full current rampup. The hybrid shutdown resistive flux consumption is $19 \mathrm{Vs}$, of which most is dissipated during the last few seconds before the current-drive system is turned on, when the xenon has been injected and $T_{e}$ is less than $1 \mathrm{eV}$. This compares to a total flux swing provided by the coils of about $700 \mathrm{Vs}$.

\section{SUMMARY OF STARTUP AND SHUTDOWN}

The PULSAR startup and shutdown scenarios marginally avoid density-driven disruptions, maintain acceptable heat fluxes at divertor plates, and avoid the generation of electron runaways. Two potential problems have been identified, but not examined in detail. The first potential problem is the possiblility of having high plasma temperatures at the divertor plate which could cause erosion and impurity generation. Advanced divertor protection schemes will have to be used during startup, beginning at rather low plasma density. The second potential problem only applies to the hybrid scenario, and is associated with the local thermal stability of a plasma with high-Z impurities and a radiation fraction of $99 \%$. Global (profile-averaged) thermal stability has been calculated, and detached, $100 \%$ radiation cases have been observed experimentally, however, more detailed study and testing of this scenario is warranted.

Summarizing, the basecase PULSAR startup scenario requires $57 \mathrm{MW}$ of auxiliary heating and $84 \mathrm{~s}$, of which 30 s are the current ramp, to reach the burn phase conditions. The startup can proceed along a thermally stable path, so that partial power operation is possible. The hybrid scenario requires $56-60$ s for startup, $60-52 \mathrm{~s}$ for shutdown, and a recharge-phase current-drive power of 70 MW of which 40-34 MW go into plasma heating. Subject to the the 200s limit on the total downtime between fusion burn pulses, the basecase has $54 \mathrm{~s}$ for recharging the transformer, and the hybrid case has about $84 \mathrm{~s}$ for recharging.

\section{REFERENCES}

1. F. Najmabadi et al., "The ARIES-I Tokamak Reactor Study," Univ. of California Los Angles report UCLA-PPG-1323 (1991).

2. C.G. Bathke, "A Comparison of Steady-State ARIES and Pulsed PULSAR Tokamak Power Plant," this meeting (1994).

3. K.A. Werley, "Reversed Field Pinch Ignition Requirements," Nuclear Fusion 31, 576 (1991).

4. D.E. Post et al., "ITER Physics," International Thermonuclear Experimental Reactor doc. series, No. 21, IAEA (1991).

5. P.I.H. Cooke, UKAEA Culham Lab., private commun. (1989).

6. K. Borrass, "Disruptive Tokamak Density Limit as Scrapeoff Layer/Divertor Phenomenon," NET Report No. 95, EUR. FU/80/89-95.

7. M. Greenwald et al., "A New Look at Density Limits in Tbkamaks," Nuclear Fusion 28,2199 (1988). 


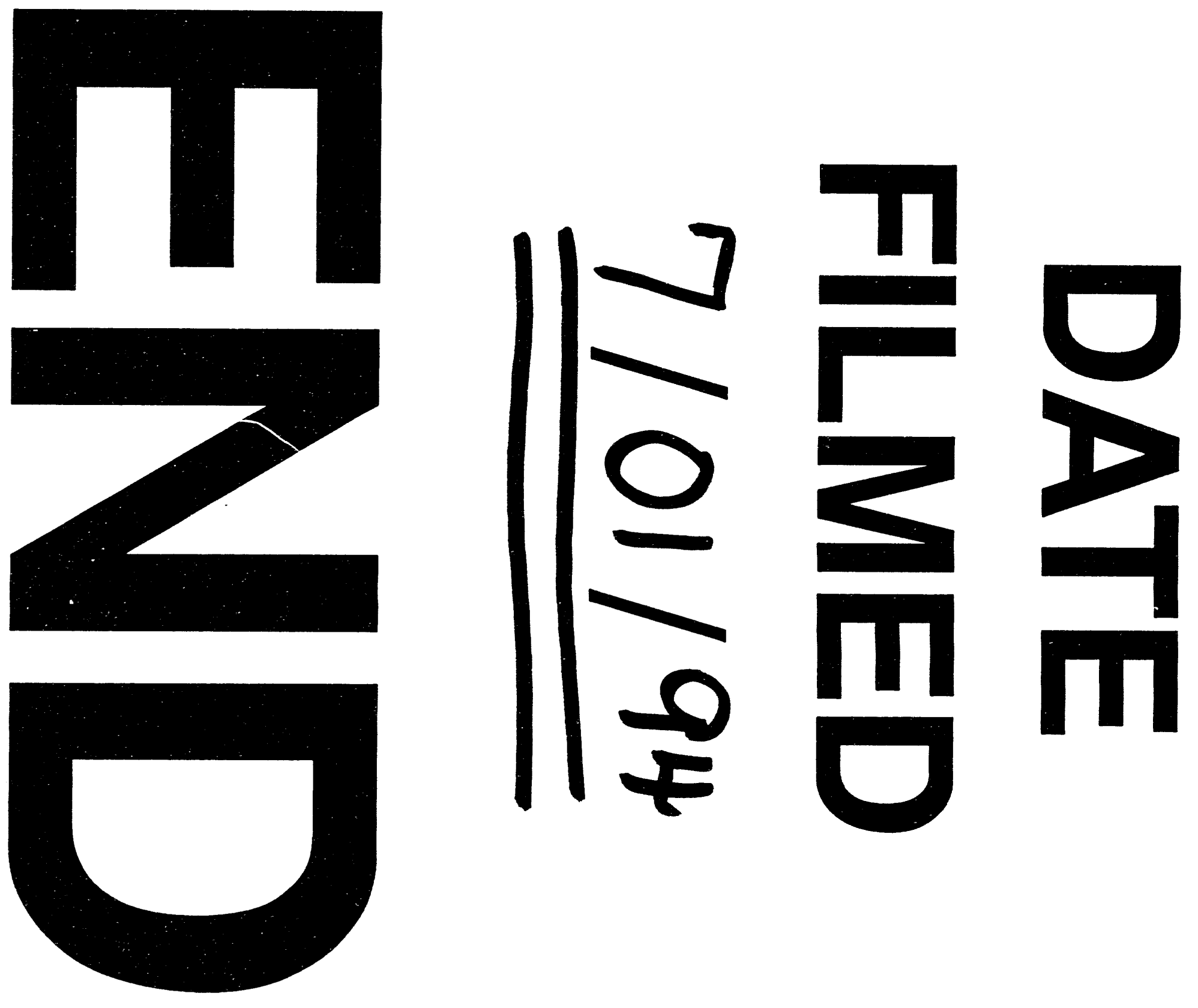


